http://dx.doi.org/10.11646/phytotaxa.152.1.6

\title{
Validation of the name Aster veitchianus (Asteraceae)
}

\author{
GUOJIN ZHANG ${ }^{1,2}$, CAIFEI ZHANG ${ }^{1,2}$, HAIHUA HU ${ }^{1,2} \&$ TIANGANG GAO ${ }^{1}$ \\ ${ }^{I}$ State Key Laboratory of Systematic and Evolutionary Botany, Institute of Botany, Chinese Academy of Sciences, Beijing 100093, \\ People's Republic of China; e-mail: Gaotg@ibcas.ac.cn (author for correspondence) \\ ${ }^{2}$ University of Chinese Academy of Sciences, Beijing 100049, People's Republic of China
}

Aster veitchianus, a species endemic to western China, is widely accepted in taxonomic and phytochemical literature (e.g. Hu 1965, Li et al. 2007, Chen et al. 2011). However, its name was not validly published, because the original diagnosis given by Ling (1935: 214) was in French, not in Latin. Thus, Ling did not validate the name A. veitchianus according to Art. 39.1 of the ICN (McNeill et al. 2012). In our survey of the taxonomic literature, we have never found this name accompanied by a Latin diagnosis or description before 31 December 2011, nor by an English diagnosis or description after 1 January 2012. We herein provide an English description to accomplish valid publication of the name. The specimen X. B. Peng 6049 (Fig. 1) deposited in PE is designated as the holotype, which was the only one specimen cited by Ling (1935).

Although he failed to validly publish this name, Ling provided a French diagnosis in 1935 (Ling 1935) and a Chinese description in F.R.P.S. (Ling 1985). His treatment was accepted and followed by some taxonomists (e.g. Li \& Shi 2007, Chen et al. 2011). After careful examination and measurement of various specimens deposited in the main herbaria of China, we conclude that A. veitchianus is morphologically uniform and easy to distinguish from its close relative A. auriculatus Franchet (1896: 379) by a lesser number of leaves and capitula, basal leaves present in anthesis, and glabrous phyllaries.

\section{Taxonomic treatment}

Aster veitchianus Hutch. \& J. R. Drumm. ex G. J. Zhang \& T. G. Gao, sp. nov.

Aster veitchianus Hutch. \& J. R. Drumm. ex Ling (1935: 214), nom. inval.

Type:-CHINA: Sichuan, Mt. E'mei, Qingyingge, alt. 950 m, 8 June 1933, X. B. Peng 6049 (holotype PE! [barcode no. 00300085]).

Description:- Perennial with creeping rhizomes. Stem erect, single, 10-40 cm tall, branched in upper part or not, villous, densely at lower part. Leaves few, less than 10; basal leaves present at anthesis, obovate, 12-90 $\times$ 6-32 mm, apex acute or obtuse, base attenuate, margin sparsely serrate (1-4 pairs), petiole $10-45 \mathrm{~mm}$ long; lower leaves oblanceolate, 30-90 × 10-30 mm, apex acute or obtuse, base attenuate, margin entire or sparsely serrate (1-4 pairs), petiole 20-40 mm long, winged, semi-amplexicaul; median leaves usually 1-2, oblanceolate, 30-65 ×5-15 mm, apex acute, base semi-amplexicaul, margin entire, sessile; bracts 1-3, narrowly lanceolate or linear, 10-30 mm long, apex acute, margin entire, sessile. Leaves adaxial face green, sparsely villous; abaxial face light green, villous on midribs, glabrous or sparsely pilose on the rest parts. Capitula 1-4, solitary or loosely corymbiform, peduncles pilose. Involucres hemispherical, 8-12 $\mathrm{mm}$ in diameter; phyllaries 2-seriate, unequal, glabrous, apex acute, purplish, margin scarious, the outer phyllaries $3-$ $5 \times 0.5 \mathrm{~mm}$, the inner $8-10 \times 0.8-1.2 \mathrm{~mm}$. Ray florets $30-40$, ligules $10-12 \mathrm{~mm}$ long, purplish, tube sparsely pilose. Disc florets 4-5 mm long, tube sparsely pilose. Achenes $1.5-2 \mathrm{~mm}$ long, oblanceolate, sparsely pilose. Pappus of barbellate bristles, whitish to slightly reddish, 2-seriate, the outer few, ca. $1 \mathrm{~mm}$ long, the inner 4-5 mm long. Flowers April-August. Fruits unknown. 
Distribution and habitat:-Aster veitchianus is found in E'mei county (Mt. E'mei) and Yinjin county, Sichuan province, China. It grows in forests and along roadsides at elevations of 800-1700 m.

Etymology and ascription:-The specific epithet "veitchianus" was originally written on a specimen in K (E. H. Wilson 4981, K) by J. Hutchinson \& J. R. Drummond, as the specimen was presented by Veitch Nurseries in the United Kingdom.

Additional specimens examined (paratypes):-CHINA. Sichuan province, E'mei county, Mt. E'mei: Anonymous 18944 (PE [barcode no. 01694265]); E. H. Wilson 4981 (K, photo); G. H. Yang 55562 (PE [barcode no. 01694263]); Wuhan University 7628 (PE [barcode no. 01694264]); 19 June 1935, T. H. Hu 207 (PE [barcode no. 00300084]); 11 July 1937, S. S. Chien 6030 (PE [barcode no. 00300080]); 17 June 1939, C. L. Sun 537 (PE [barcode no. 00300081]); 4 June 1941, W. P. Fang 16837 (PE [barcode no. 01776712]); 29 May 1956, S. Z. Yu 49540 (SZ, photo [barcode no. 00265612]); 23 April 1957, Anonymous 54165 (PE [barcode no. 00300078]); 28 April 1957, G. H. Yang 55562 (PE [barcode nos. 01694263, 00300079]; SZ, photo [barcode no. 00265480]); 16 May 1957, Y. X. Xiao 30 (SZ, photo [barcode no. 00265649]); 20 May 1957, G. H. Yang 54653 (PE [barcode no. 00300082]); 29 May 1957, G. H. Yang 54953 (PE [barcode no. 00300083]); 1 June 1964, Y. L. Chen 011 (PE [barcode no. 00300075]); 3 June 1964, Y. L. Chen 017 (PE [barcode nos. 00300091, 00300092, 00300093]); 25 August 1964, K. J. Guan, J. W. Wang \& C. L. Li 1717 (PE [barcode nos. 00300076, 00300077]); 7 June 1980, Z. Y. Zhang et al. 876 (PE [barcode no. 01824645]). Sichuan province, Yinjin county: 8 July 1938, Z. W. Yao 2252 (PE [barcode no. 00300087]); 22 June 1939, Z. W. Yao 3795 (PE [barcode no. 00300086]).

Identification key to Aster veitchianus and A. auriculatus

1. Leaves less than 10 , basal ones present at anthesis, the others at lower part of the stem, abaxial face not glandular; capitula 1-4; phyllaries glabrous A. veitchianus

- Leaves more than 15 , basal ones withering at anthesis, the others at median and upper part of the stem, abaxial face glandular; capitula more than 10; phyllaries glandular A. auriculatus

\section{Acknowledgements}

The authors thank the curators of the herbaria PE, $K \&$ SZ for access to specimens and providing images. This study was funded by National Natural Science Foundation of China, grant no. 31270237.

\section{References}

Chen, Y.L., Brouillet, L. \& Semple, J.C. (2011) Aster. In: Wu, Z.Y. \& Raven, P.H. (eds.) Flora of China 20-21. Science Press, Beijing \& Missouri Botanical Garden Press, St. Louis, pp. 574-632.

Franchet, M.A. (1896) Compositae Novae e Flora Sinensi (suite). Journal de Botanique 10: 377-386.

Hu, S.Y. (1965) The Compositae of China (II). Quarterly Journal of the Taiwan Museum 18: 233-333.

Li, Z.Y. \& Shi, L. (2007) Plants of Mount Emei. Beijing Science and Technology Press, Beijing, 417 pp.

Li, E.W., Gao, X., Gao, K. \& Jia, Z.J. (2007) Labdane Diterpenoid Glycosides from Aster veitchianus. Chemistry \& Biodiversity 4: 531-538. http://dx.doi.org/10.1002/cbdv.200790046

Ling, Y. (1935) Les Composées Chinoises de l'Herbier de l'Academie de Peiping (suite). Contributions from the Institute of Botany, National Academy of Peiping 3: 123-220.

Ling, Y. (1985) Aster. In: Ling, Y. \& Chen, Y.L. (eds.) Flora Reipublicae Popularis Sinicae 74. Science Press, Beijing, pp. 133-254.

McNeill, J., Barrie, F.R., Buck, W.R., Demoulin, V., Greuter, W., Hawksworth, D.L., Herendeen, P.S., Knapp, S., Marhold, K., Prado, J., Prud'homme van Reine, W.F., Smith, G.F., Wiersema, J.H. \& Turland, N.J. (eds.) (2012) International Code of Nomenclature for algae, fungi, and plants (Melbourne Code) adopted by the Eighteenth International Botanical Congress Melbourne, Australia, July 2011 [Regnum Vegetabile 154]. Gantner, Ruggell, $\mathrm{XXX}+208 \mathrm{pp}$. 